\title{
Reconocimiento de Patrones Vocálicos mediante la implementación de una red Neuronal Artificial Utilizando Sistemas Embebidos
}

\author{
Olga L. Ramos ${ }^{(1)}$, Diego A. Rojas ${ }^{(1)}$ y Jorge E. Saby(2) \\ (1) Grupo de Aplicaciones Virtuales GAV, Facultad de Ingeniería, Universidad Militar Nueva Granada, \\ Carrera 11 \# 101-80, Bogotá, Colombia. (e-mail: olga.ramos@unimilitar.edu.co; \\ u1801620@unimilitar.edu.co) \\ (2) GICOECOL, Facultad de Ingeniería, Universidad Distrital Francisco José de Caldas, Carrera 7 No. $40 \mathrm{~B}$ - \\ 53, Bogotá, Colombia. (e-mail: jesabyb@udistrital.edu.co)
}

Recibido Feb. 25, 2016; Aceptado Abr. 18, 2016; Versión final Abr. 28, 2016, Publicado Oct. 2016

\begin{abstract}
Resumen
En este trabajo se presentan los resultados del diseño y desarrollo de una red neuronal de tipo feed-forward en un sistema embebido, para identificar patrones asociados con la pronunciación de fonemas vocálicos del idioma español. Para el entrenamiento de la red fueron utilizados los coeficientes cepstrales de las frecuencias de Mel (MFCC), extraídos a partir de señales de audio provenientes de la pronunciación de las vocales abiertas $(/ \mathrm{a} / \mathrm{/}, \mathrm{e} / \mathrm{y} / \mathrm{o} /)$. La captura de nuevas muestras se realizó a través de un computador y un sistema embebido para la evaluación, comprobación y comparación del desempeño de la red neuronal. Se obtuvo un porcentaje de identificación correcta por encima del 98\%. Esto indica que el error en la separación de clases fue inferior al $2 \%$ en la red neuronal al momento de evaluar patrones asociados a alguna de las tres vocales. Basado en los resultados obtenidos se concluye que la implementación de algoritmos de inteligencia artificial para tareas de clasificación en sistemas embebidos es factible, y presenta resultados similares a los que tendría el mismo sistema operando con los recursos de un computador.
\end{abstract} del habla

\section{Recognition of Vowel Patterns by Implementing an Artificial Neural Network in an Embedded System}

\begin{abstract}
In this work the results of the design and development of a neural network type feed-forward in an embedded system, to identify patterns associated with the pronunciation of vowels of Spanish language are presented. For the training of the network Mel frequencies cepstrals coefficients extracted from audio signals related to the pronunciation of the open vowels $(/ \mathrm{a} / \mathrm{l} / \mathrm{e} /$ and $/ \mathrm{o} /)$ were used. The capture of new samples was performed by using a computer and an embedded system to evaluate, test and compare the performance of the neural network. A percentage of correct identification above $98 \%$ was obtained indicating that the error in the class separation was less than $2 \%$ for patterns obtained from any of the three vowels. Based on the results it is concluded that implementation of artificial intelligence algorithms for classification tasks in embedded systems is feasible, and presents similar results to those that would have the same system working with the resources of a computer.
\end{abstract}

Keywords: neural networks; audio signals; embedded system; cepstrals coefficients; speech recognition 


\section{INTRODUCCIÓN}

La comunicación es una herramienta de uso cotidiano para el ser humano, que le permite desenvolverse en un ambiente especifico, y puede dividirse en comunicación verbal (Gärdenfors y Warglien, 2013), que se refiere a la divulgación de información a través de signos lingüísticos característicos de una cultura, y pueden ser orales o escritos. Por otro lado, se encuentra la comunicación no verbal (Knapp, et al., 2013), que es la transmisión de mensajes sin el uso de palabras, es decir, indicios, gestos, o señales como el lenguaje facial o corporal. De las dos categorías anteriores, el tipo de comunicación más común es el habla, que está basada en la combinación de léxico y nombres extraídos de un grupo de palabras denominado vocabulario. La producción del habla, a nivel lingüístico describe como se ubican los labios, mandíbula y lengua para lograr emitir un sonido, y depende de dos procesos fundamentales, la percepción, por medio de la cual se interpretan y entienden los sonidos propios de un lenguaje, y la repetición, que es cuando un sonido se trasforma en un movimiento inconsciente volviendo el habla un movimiento mecánico.

Estos procesos, han sido de gran interés en las últimas décadas para la comunidad científica, la cual a partir de los últimos desarrollos tecnológicos han hecho de las técnicas de procesamiento digital de señales métodos más eficientes y eficaces para elaborar algoritmos de tratamiento de voz cada vez más complejos, como se evidencia en el trabajo realizado por (Brandão, et al., 2012), donde elaboran un modelo 3D del tracto vocal para analizar la propagación de las ondas sonoras en el mismo, y en (Martínez, et al., 2010) buscan realizar la codificación y decodificación de señales de voz utilizando bancos de filtro multi-resolución, con el fin de conseguir un método para el ocultamiento de información.

Otro ejemplo de lo anterior es el trabajo realizado en (Mitra, et al., 2014), en éste desarrollaron un sistema para identificar fonemas del idioma Árabe, mediante modulación de coeficientes cepstrales alcanzando una tasa de reconocimiento igual a $21.6 \%$ en ambientes con gran cantidad de distorsión y perturbaciones, igualmente en (Adam, et al., 2013) fue elaborado un sistema para reconocimiento automático de habla (RAH) capaz de identificar las 26 vocales del idioma inglés, utilizando coeficientes cepstrales calculados a través de la transformada Wavelet discreta, logrando un reconocimiento promedio de $67 \%$.

También han sido elaborado trabajos para aumentar el desempeño de los sistemas de interpretación automática de habla en ambientes ruidosos, como lo muestra (Yoshioka, et al.., 2012), en una revisión de las técnicas o métodos utilizados para tratar los problemas de reverberación en el procesamiento de señales de audio, con el fin de mejorar la capacidad de reconocimiento en máquinas ubicadas en ambientes con reflexión de las ondas acústicas, además se han realizado avances en tareas de compresión de datos en señales de audio, para reducir la dimensión de los datos y mejorar tareas relacionadas con la extracción de características, como se muestra en (Moreno, et al., 2014).

Con la llegada de las técnicas de inteligencia artificial para clasificación de patrones, se han llevado a cabo desarrollos como los mostrados en (Lu, et al., 2010), donde utilizan redes neuronales auto-ajustables y modelos ocultos de Markov para alcanzar un porcentaje de acierto, en el reconocimiento de patrones asociados al habla, superior al $90 \%$, y en (Ting, et al., 2013) muestran una metodología basada en dos tipos de redes neuronales, redes neuronales de pulso acoplado y redes neuronales probabilísticas obteniendo como mejor resultado un $95 \%$ de acierto, teniendo en cuenta los datos adquiridos de la pronunciación de los números de cero a nueve para el idioma inglés. Por otro lado, en (Siniscalchi, et al., 2014) se hace una revisión del estado de arte relacionado al uso de redes neuronales para sistemas de reconocimiento de habla, a través de diferentes técnicas de extracción de características y diferentes tipos de reconocimiento.

Un importante avance en los desarrollos elaborados con inteligencia artificial involucra los sistemas embebidos, como se aprecia en (Bao, et al., 2012), donde utilizan una red neuronal programada en una tarjeta de desarrollo que cuenta con un microcontrolador ARM 9 para realizar el reconocimiento de caminos que permita realizar tareas de exploración con un robot móvil, de la misma forma en (Chih-Lyang Hwang, et al., 2015) y (Chiang, et al., 2011), se muestran los resultados de utilizar redes neuronales para visión de máquina y posicionamiento de máquinas en entornos delimitados respectivamente, por último, en el trabajo desarrollado en (Baek, et al., 2008) se utiliza una red neuronal embebida para controlar un sistema de alimentación hibrido.

Teniendo en cuenta todo lo mencionado anteriormente, en este trabajo se presentan los resultados de extraer características a partir de señales de audio provenientes del habla, específicamente de la producción de las vocales /a/, /e/ y /o/ a través de la técnica de MFCC. De la misma manera se realiza la clasificación y reconocimiento de los patrones obtenidos con el cálculo de los coeficientes, utilizando un algoritmo de aprendizaje de máquina basado en redes neuronales, el cual también fue implementado dentro de un sistema embebido, con el fin de comparar su desempeño cuando éste opera utilizando los recursos de un ordenador y los del sistema embebido. La segunda sección de éste documento contiene la 
descripción de la metodología utilizada para el desarrollo del sistema propuesto. En la tercera sección se resumen y analizan los resultados y por último, se presentan las conclusiones obtenidas con el desarrollo de esta investigación, exponiendo una perspectiva futura para continuar el desarrollo de este tipo de tecnologías.

\section{MATERIALES Y MÉTODOS.}

Para desarrollar el sistema de reconocimiento, se llevaron a cabo los procesos mostrados en el esquema de la figura 1. En este diagrama se incluyen las etapas de adquisición de señales a través del sistema embebido y un computador, el procesamiento de las mismas, utilizando segmentación y algoritmia de extracción de características, como los coeficientes cepstrales de las frecuencias de Mel (MFCC) que fueron utilizados para el entrenamiento de la red y por lo tanto en la tarea de clasificación. Por último se verificó la respuesta de la red ante un patrón nuevo, obteniendo una respuesta en términos de probabilidad, que indica en porcentaje el grado de pertenencia que una nueva muestra presenta con una clase o grupo.

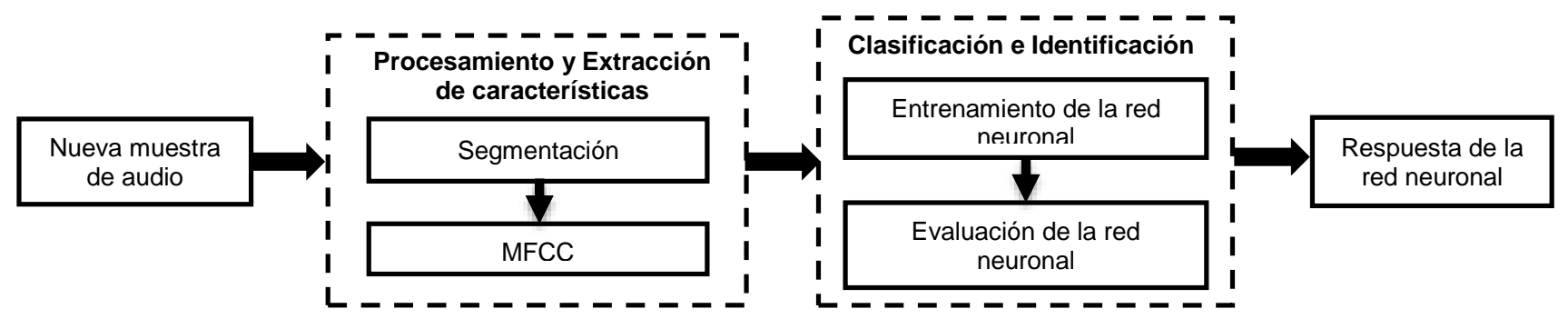

Fig 1: Diagrama de la metodología propuesta.

Siguiendo la metodología expuesta en la figura 1, se contó con un grupo de 10 hablantes, todos del género masculino y una edad promedio de 23 años, de quienes fueron grabadas 3 muestras por cada unidad fonológica que se quería reconocer, siguiendo la metodología utilizada por (Mella y Soto-Barba, 2005) y (Valverde y Soto-Barba, 2015), para elaborar estudios sobre las variaciones de los formantes vocálicos para hablantes cuya segunda lengua es el español, y la pronunciación de palabras acentuadas en hablantes hispanos de Chile, se utilizaron muestras de 8 y 6 hablantes respectivamente para caracterizar el comportamiento de los formantes vocálicos.

Las muestras fueron adquiridas a través de la tarjeta de audio de un ordenador, cuya frecuencia de muestreo era de $8 \mathrm{kHz}$, el grupo de datos obtenido fue utilizado para la etapa de procesamiento y la implementación de los coeficientes cepstrales de las frecuencias de Mel, que fue la técnica escogida para realizar la extracción de características. Los MFFC calculados se utilizaron como material de entrenamiento y validación de la red neuronal, para la posterior programación de la misma en el sistema embebido, y de esta manera volver la red neuronal un algoritmo portable.

El sistema embebido contaba con un microcontrolador de 32 bits ARM Cortex-M4 de la serie STM32F4 cuya velocidad máxima de procesamiento es $168 \mathrm{MHz}$, cuenta con una memoria Flash de 1 Mbyte, y una memoria de acceso aleatorio de $192 \mathrm{Kbyte,} \mathrm{el} \mathrm{conversor} \mathrm{análogo-digital} \mathrm{tiene} \mathrm{una} \mathrm{resolución} \mathrm{de} \mathrm{10-bit} \mathrm{y} \mathrm{fue}$ configurado para adquirir 8000 muestras por segundo, debido a que las señales de voz cuentan con información relevante hasta los $4 \mathrm{Khz}$, de allí que los sistema de telefonía fija solamente tiene en cuenta los primeros $3.8 \mathrm{KHz}$ de la señal.

\section{COEFICIENTES CEPSTRALES DE LAS FRECUENCIAS DE MEL (MFCC)}

A pesar del gran número de métodos, procedimientos y técnicas para extraer características relevantes de señales de audio (Fu, et al., 2012; Kuamr, et al., 2014; Yusnita, et al., 2011), el computo de los MFCC ha mostrado ser una de las herramientas más utilizadas para procesas ese tipo de señales, debido a su robustez y calidad en las características que entrega. En la figura 2 se muestran los procesos más importantes para lograr la obtención de MFCC para una señal de voz.

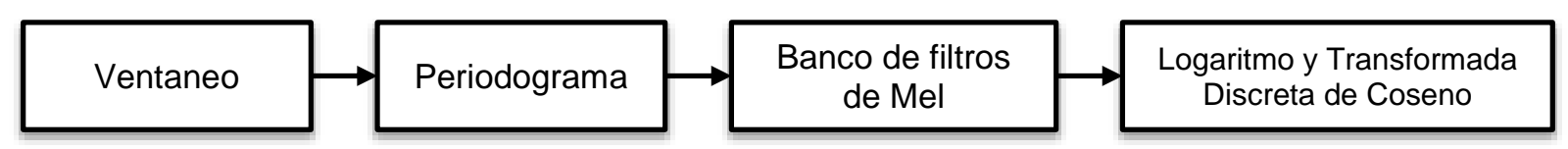

Fig 2: Procesos involucrados en el cálculo de los MFCC. 
Antes del proceso de ventaneo, se debe realizar una operación de pre-procesamiento conocida como preénfasis, ésta consiste en aplicar un filtro pasa alto de primer orden para incrementar la cantidad de energía presente en las altas frecuencias de la señal, y hacer de éste un parámetro relevante en las posteriores etapas del procesamiento. Una vez terminado el preénfasis, se realizó el ventaneo de la señal, dividiéndola en tramos de $25 \mathrm{~ms}$, obteniendo así 8 ventanas, debido a que la información más relevante de la señal tenía un intervalo de $0.2 \mathrm{~s}$. Para obtener la cantidad de energía presente en cada frecuencia o periodograma de la señal, se aplicó la transformada de Fourier a cada uno de los segmentos obtenidos previamente como se ilustra en la ecuación 1.

$P_{i}[k]=\frac{1}{N}\left|\sum_{n=1}^{N} x_{i}[n] h[n] e^{-j 2 \pi k n / N}\right|^{2}$

En esta ecuación, $x_{i}[n]=$ Segmento de la señal; $h[n]=$ Ventana tipo Hamming; y $N=$ Número total de muestras.

Después de hallar el periodograma de la señal, se debe filtrar el conjunto de datos resultantes utilizando un banco de filtros de Mel (Kuan, et al., 2014) y por último se aplican las operaciones de logaritmo y transformada discreta de coseno. Como es descrito en (Yuan, et al., 2015), se obtuvieron 13 coeficientes debido a que la cantidad de filtros de Mel que debe ser usada para cubrir el rango de frecuencias de la señal es aproximadamente 13, no obstante, el primer coeficiente indica la cantidad de energía presente en la señal, que es un valor poco relevante al momento de determinar la vocal, debido a que dos vocales pueden tener un valor similar de energía en términos de amplitud. Con lo dicho anteriormente, se obtuvieron 12 coeficientes por ventana, dando un total de 96 coeficientes por cada señal de voz registrada.

\section{RED NEURONAL}

Para la tarea de reconocimiento se utilizó una red neuronal de tipo feed-forward, la cual consta de tres capas, una primera que es la capa de entrada para ingresar los datos de las características a la red, la segunda es la capa oculta, que se encarga de la mayor parte del procesamiento, y por último se tiene la capa de salida para ajustar los datos provenientes del cálculo elaborado en la capa oculta. La topología o arquitectura de la red escogida es como se muestra en la figura 3.

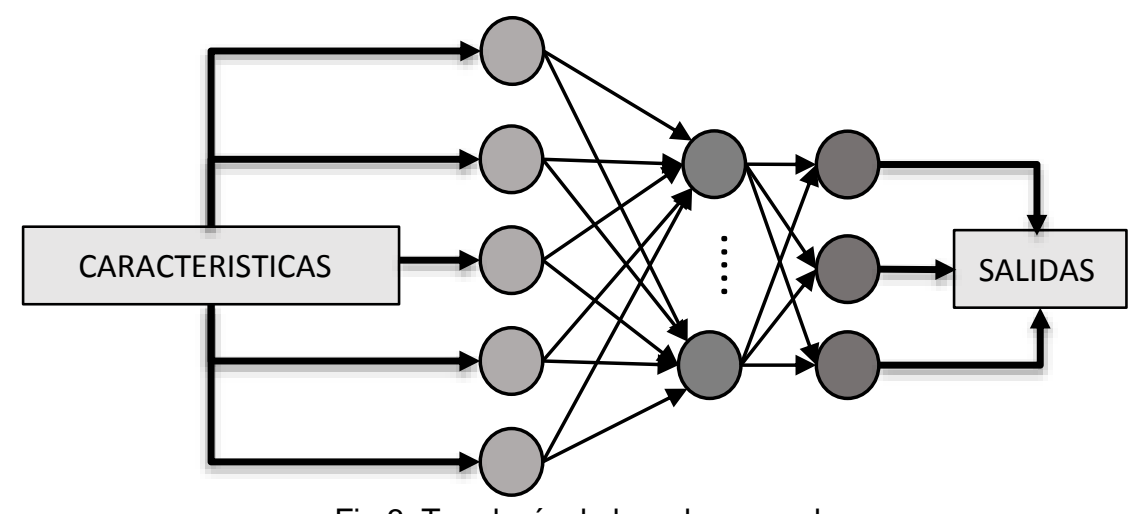

Fig 3: Topología de la red neuronal

El entrenamiento se llevó a cabo utilizando el algoritmo retro propagación junto con el error por entropía cruzada, mostrado en la ecuación 2, como valor de actualización para los pesos.

Error $=-\frac{1}{N} \sum_{n=1}^{N}\left[y_{n} \log \left(\hat{y}_{n}\right)+\left(1-y_{n}\right) \log \left(1-\hat{y}_{n}\right)\right]$

El entrenamiento en su totalidad fue realizado antes de ingresar la estructura de la red en el sistema embebido.

\section{IMPLEMENTACIÓN EN EL SISTEMA EMBEBIDO}

El proceso de embeber la red fue dividido en dos etapas, de las cuales la primera tuvo en consideración los valores de entrada, pesos y bias de la capa oculta, así como la función de activación tangente sigmoidal como se muestra en la figura 4. Aunque existen otras funciones de activación, como la lineal o logarítmica sigmoidal (logsig), se utilizó la tangente sigmoidal porque permite obtener valores positivos y negativos entre 
-1 y 1, aproximándose al comportamiento que presentan los coeficientes cepstrales de las frecuencias de Mel y al mismo tiempo satura los valores obtenidos en la capa oculta.

Las funciones logsig y lineal, no permiten el uso de valores negativos, y no saturan los valores de salida respectivamente, inconvenientes que dificultan el entrenamiento de la red, debido a que no contemplan en su totalidad el comportamiento de los patrones, lo que lleva a una mala agrupación de los datos, o demorar el tiempo de entrenamiento al aumentar o disminuir abruptamente el valor de los pesos. A continuación se describen los procesos utilizados para programar la red neuronal en el sistema embebido.

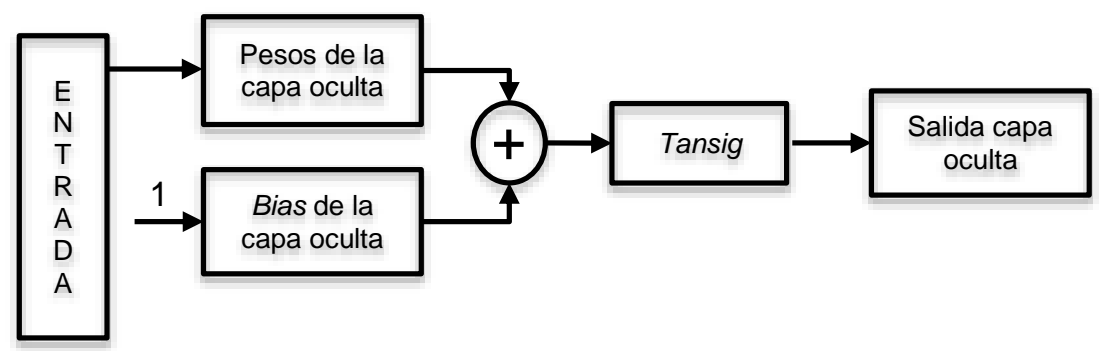

Fig 4. Diagrama de bloques para la capa oculta

Para programar el proceso mostrado en la figura 4 en el sistema embebido se utilizaron las matrices, vectores y operaciones presentadas en la ecuación 3.

$\left[\begin{array}{c}H L o_{1} \\ \vdots \\ H L o_{m}\end{array}\right]=A F_{H L}\left(\left[\begin{array}{ccc}W h l_{1,1} & \cdots & W h l_{1, n} \\ \vdots & \ddots & \vdots \\ W h l_{m, 1} & \cdots & W h l_{m, n}\end{array}\right]\left[\begin{array}{c}I_{1} \\ \vdots \\ I_{n}\end{array}\right]+\left[\begin{array}{c}B h l_{1} \\ \vdots \\ B h l_{m}\end{array}\right]\right)$

En esta ecuación: $I=$ Entrada de la red neuronal; $n=$ Número de patrones de entrada; $W h l=$ Pesos de la capa oculta; $B h l=$ Bias de la capa oculta; $H L o=$ Salida de la capa oculta; $m=$ Número de neuronas de la paca oculta; $A F_{H L}=$ Función de activación para la capa oculta, específicamente la función tangente sigmoide, cuya expresión se muestra en la ecuación

$\operatorname{Tansig}(x)=\frac{2}{1+e^{-2 x}}-1$

Para embeber el proceso anterior se debe definir un vector columna de tamaño $m$ para almacenar los valores calculados por la capa oculta de la red neuronal. Es necesario definir la matriz de pesos con un tamaño de $m \times n$, para multiplicarla por el vector columna de entrada, cuyo tamaño es $n$, posteriormente se suma el vector con los valores de bias y finalmente se aplica la función de activación tangente sigmoidal a cada uno de los componentes del vector resultante, obteniendo así la salida de la capa oculta. La segunda parte involucra los valores provenientes de la capa oculta para realizar la implementación de la capa de salida como se muestra en la figura 5 , siguiendo el mismo procedimiento explicado para programar la capa oculta.

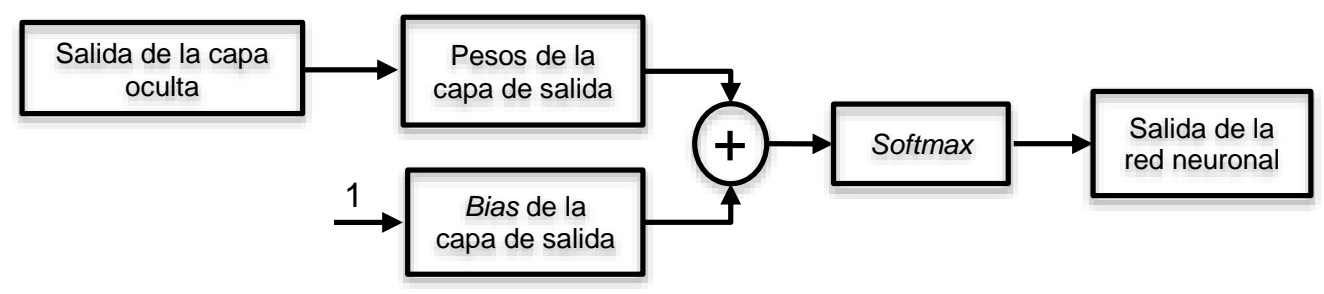

Fig 5: Diagrama de bloques para la respuesta de la capa oculta y la capa de salida de la red neuronal.

En este diagrama se tienen en cuenta los valores de los pesos y bias calculados para el funcionamiento de la capa de salida, así como la programación de la función exponencial normalizada o softmax para finalizar la tarea de clasificación. El uso de la función softmax permite normalizar la salida de la red, además de entregar un valor probabilístico entre 0 y 1 , de acuerdo a la distribución de los valores obtenidos para cada una de las clases o grupos. La expresión mostrada en la ecuación 5 , resume las operaciones necesarias para que la red neuronal funcione de manera adecuada en el sistema embebido. 
$\left[\begin{array}{c}N N o_{1} \\ \vdots \\ N N o_{s}\end{array}\right]=A F_{O L}\left(\left[\begin{array}{ccc}W o l_{1,1} & \cdots & W^{\prime} l_{1, m} \\ \vdots & \ddots & \vdots \\ W o l_{s, 1} & \cdots & W o l_{s, m}\end{array}\right]\left[\begin{array}{c}H L o_{1} \\ \vdots \\ H L o_{s}\end{array}\right]+\left[\begin{array}{c}\text { Bol }_{1} \\ \vdots \\ \text { Bol }_{s}\end{array}\right]\right)$

Aquí: $W o l=$ Pesos de la capa de salida; $m=$ Número de neuronas en la capa oculta; $B o l=B i a s$ de la capa de salida; $N N o=$ Salida y respuesta de la red neuronal; $s=$ Número de neuronas en la capa de salida; $A F_{O L}=$ Función de activación de la capa oculta, conocida como exponencial normalizada y se expresa en la ecuación 6.

$\operatorname{Softmax}(x)=\frac{e^{x}}{\sum_{k=1}^{k=N} e^{k}}$

\section{RESULTADOS}

Para el caso expuesto en este documento, se desarrolló un sistema de reconocimiento de patrones, dedicado a la identificación de tres unidades fonológicas del idioma español de tipo abierto $(/ \mathrm{a} /, \mathrm{e} / \mathrm{e}$ y /o/). Como primer resultado, se realizó la configuración y entrenamiento de 18 redes neuronales con diferentes cantidades de neuronas en la capa oculta. En la capa de entrada, se utilizaron 96 neuronas para ingresar los valores de los coeficientes obtenidos en la fase de extracción de características, y en la capa de salida fueron programadas tres neuronas, una por cada clase o categoría que se quería identificar. En la figura 6 , se ilustra el comportamiento que mostró el error de entrenamiento a medida que se iban aumentando la cantidad de neuronas en la capa oculta.

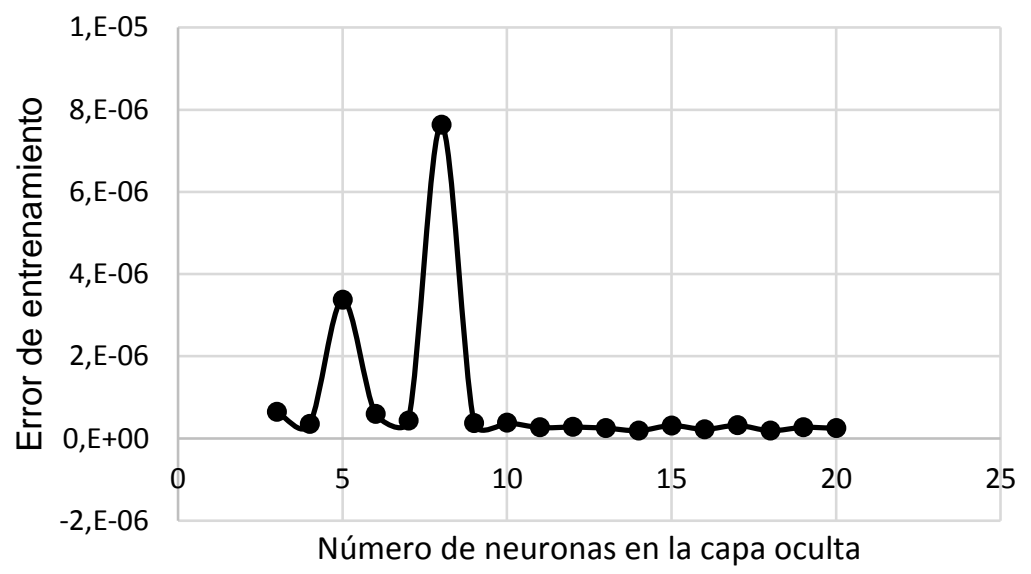

Fig 6: Error de entrenamiento para la red neuronal.

De acuerdo a los datos presentados en la figura 6 , se escogió el valor de 7 neuronas para realizar la implementación en el sistema embebido, lo anterior obedece a que el comportamiento de la red no mejoró significativamente para valores de neuronas superiores al escogido. En la figura 7, se puede observar el proceso de entrenamiento de la red neuronal, el cual necesitó 29 épocas para alcanzar un valor lo suficientemente bajo para detener el aprendizaje.

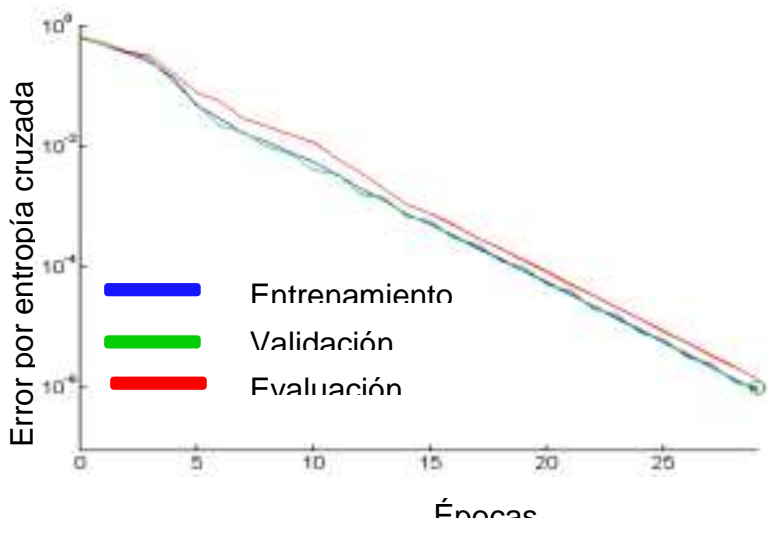

Fig 7: Comportamiento de la red neuronal durante el entrenamiento. Error de entrenamiento mínimo igual a 9,86×10-7 
En la tabla 1, se encuentran los resultados de la evaluación de la red neuronal entrenada off-line y la que se implementó el sistema embebido, para 10 patrones provenientes del procesamiento de señales de audio con información de la vocal /a/. Para las muestras de la vocal/a/, el sistema de reconocimiento respondió de manera adecuada, consiguiendo una tasa de reconocimiento promedio igual a $99.43 \%$ para la red entrenada off-line y $98.715 \%$ para la red embebida, teniendo en cuenta los datos obtenidos para las 10 muestras. En esta tabla cada columna es el porcentaje de pertenencia que la red asigna a cada nueva muestra. Por ejemplo, en el caso de la muestra número 1, que proviene de registrar la pronunciación de la vocal /a/, la red asigna una pertenencia del $99.38 \%$ con respecto al grupo 1, 1,86×10-05\% para el grupo 2 y $2,80 \times 10^{-05 \%}$ para el grupo 3 en el sistema embebido, con lo cual se puede afirmar que el nuevo patrón pertenece al grupo 1, que fue entrenado con los MFCC extraídos de las adquiridas al pronunciar la vocal /a/.

Tabla 1. Porcentajes de pertenencia para nuevas muestras de la vocal /a/

\begin{tabular}{|c|c|c|c|c|c|c|}
\hline \multirow{2}{*}{ \# Muestra } & \multicolumn{3}{|c|}{$\begin{array}{c}\text { Porcentaje de pertenencia con la } \\
\text { red neuronal en el computador (\%) }\end{array}$} & \multicolumn{2}{c|}{$\begin{array}{c}\text { Porcentaje de pertenencia con la } \\
\text { red neuronal embebida (\%) }\end{array}$} \\
\cline { 2 - 7 } & $\begin{array}{c}\text { Clase 1: } \\
\text { Vocal /a/ }\end{array}$ & $\begin{array}{c}\text { Clase 2: } \\
\text { Vocal /e/ }\end{array}$ & $\begin{array}{c}\text { Clase 3: } \\
\text { Vocal /o/ }\end{array}$ & $\begin{array}{c}\text { Clase 1: } \\
\text { Vocal /a/ }\end{array}$ & $\begin{array}{c}\text { Clase 2 : } \\
\text { Vocal /e/ }\end{array}$ & $\begin{array}{c}\text { Clase 3: } \\
\text { Vocal /o/ }\end{array}$ \\
\hline 1 & 99,38 & $1,86 \times 10^{-05}$ & $2,80 \times 10^{-05}$ & 99,21 & $1,89 \times 10^{-04}$ & $5,92 \times 10^{-04}$ \\
\hline 2 & 99,57 & $2,13 \times 10^{-05}$ & $2,62 \times 10^{-05}$ & 100 & $7,62 \times 10^{-05}$ & $1,44 \times 10^{-04}$ \\
\hline 3 & 99,43 & $2,78 \times 10^{-05}$ & $2,94 \times 10^{-05}$ & 99,78 & $8,67 \times 10^{-04}$ & $1,31 \times 10^{-03}$ \\
\hline 4 & 99,54 & $1,75 \times 10^{-05}$ & $2,88 \times 10^{-05}$ & 94,27 & $1,05 \times 10^{-04}$ & $5,71 \times 10^{-02}$ \\
\hline 5 & 99,52 & $2,11 \times 10^{-05}$ & $2,67 \times 10^{-05}$ & 99,75 & $3,87 \times 10^{-04}$ & $2,32 \times 10^{-01}$ \\
\hline 6 & 99,15 & $1,74 \times 10^{-05}$ & $2,91 \times 10^{-05}$ & 99,04 & $2,13 \times 10^{-04}$ & $2,53 \times 10^{-03}$ \\
\hline 7 & 99,56 & $1,73 \times 10^{-05}$ & $3,11 \times 10^{-05}$ & 99,74 & $4,41 \times 10^{-05}$ & $1,21 \times 10^{-03}$ \\
\hline 8 & 99,79 & $1,72 \times 10^{-05}$ & $2,87 \times 10^{-05}$ & 99,52 & $1,45 \times 10^{-04}$ & $7,95 \times 10^{-04}$ \\
\hline 9 & 99,34 & $1,77 \times 10^{-05}$ & $4,90 \times 10^{-05}$ & 98,42 & $2,31 \times 10^{-04}$ & $5,23 \times 10^{-05}$ \\
\hline 10 & 99,12 & $1,73 \times 10^{-05}$ & $2,96 \times 10^{-05}$ & 97,42 & $3,15 \times 10^{-04}$ & $6,43 \times 10^{-04}$ \\
\hline Promedio & 99,44 & $1,73 \times 10^{-05}$ & $3,07 \times 10^{-05}$ & 98,715 & $2,57 \times 10^{-04}$ & $2,96 \times 10^{-02}$ \\
\hline
\end{tabular}

De igual manera, en la tabla 2 se encuentran los datos relacionados con la identificación de los patrones asociados a la pronunciación de la vocal /e/ utilizando los MFCC, alcanzando un porcentaje de clasificación promedio igual a $99.417 \%$ con el entrenamiento off-line y $98.972 \%$ en el sistema embebido. Lo anterior indica que la separación de clases para los datos relacionados con la captura de la señal proveniente de pronunciar la vocal/e/ es lo suficientemente amplia para separar al menos dos vocales.

Tabla 2. Porcentajes de pertenencia para nuevas muestras de la vocal /e/

\begin{tabular}{|c|c|c|c|c|c|c|}
\hline \multirow{2}{*}{ \# Muestra } & \multicolumn{3}{|c|}{$\begin{array}{c}\text { Porcentaje de pertenencia con la } \\
\text { red neuronal en el computador (\%) }\end{array}$} & \multicolumn{2}{c|}{$\begin{array}{c}\text { Porcentaje de pertenencia con la } \\
\text { red neuronal embebida (\%) }\end{array}$} \\
\cline { 2 - 7 } & $\begin{array}{c}\text { Clase 1: } \\
\text { Vocal /a/ }\end{array}$ & $\begin{array}{c}\text { Clase 2: } \\
\text { Vocal /e/ }\end{array}$ & $\begin{array}{c}\text { Clase 3: } \\
\text { Vocal /o/ }\end{array}$ & $\begin{array}{c}\text { Clase 1: } \\
\text { Vocal /a/ }\end{array}$ & $\begin{array}{c}\text { Clase 2 : } \\
\text { Vocal /e/ }\end{array}$ & $\begin{array}{c}\text { Clase 3: } \\
\text { Vocal /o/ }\end{array}$ \\
\hline 1 & $4,18 \times 10^{-05}$ & 99,37 & $8,51 \times 10^{-10}$ & $4,07 \times 10^{-05}$ & 98,64 & $8,83 \times 10^{-09}$ \\
\hline 2 & $4,92 \times 10^{-05}$ & 99,45 & $8,57 \times 10^{-10}$ & $4,02 \times 10^{-05}$ & 98,88 & $1,01 \times 10^{-09}$ \\
\hline 3 & $4,08 \times 10^{-05}$ & 99,43 & $8,52 \times 10^{-10}$ & $3,88 \times 10^{-05}$ & 99,83 & $1,30 \times 10^{-09}$ \\
\hline 4 & $4,16 \times 10^{-05}$ & 99,42 & $8,54 \times 10^{-10}$ & $3,43 \times 10^{-05}$ & 99,72 & $3,19 \times 10^{-09}$ \\
\hline 5 & $4,36 \times 10^{-05}$ & 99,36 & $9,47 \times 10^{-10}$ & $3,92 \times 10^{-05}$ & 99,38 & $1,32 \times 10^{-09}$ \\
\hline 6 & $4,64 \times 10^{-05}$ & 99,43 & $8,56 \times 10^{-10}$ & $3,84 \times 10^{-05}$ & 98,75 & $2,31 \times 10^{-09}$ \\
\hline 7 & $4,03 \times 10^{-05}$ & 99,41 & $8,51 \times 10^{-10}$ & $5,21 \times 10^{-05}$ & 99,75 & $4,32 \times 10^{-09}$ \\
\hline 8 & $4,56 \times 10^{-05}$ & 99,43 & $8,50 \times 10^{-10}$ & $4,05 \times 10^{-05}$ & 98,45 & $5,62 \times 10^{-09}$ \\
\hline 9 & $4,37 \times 10^{-05}$ & 99,45 & $8,74 \times 10^{-10}$ & $3,85 \times 10^{-05}$ & 99,54 & $5,47 \times 10^{-09}$ \\
\hline 10 & $4,62 \times 10^{-05}$ & 99,42 & $8,51 \times 10^{-10}$ & $4,52 \times 10^{-05}$ & 96,78 & $2,21 \times 10^{-09}$ \\
\hline Promedio & $4,39 \times 10^{-05}$ & 99,417 & $8,64 \times 10^{-10}$ & $4,08 \times 10^{-05}$ & 98,972 & $3,56 \times 10^{-09}$ \\
\hline
\end{tabular}

Por último, en la tabla 3 se resumen los datos obtenidos al evaluar la red neuronal con los coeficientes calculados al aplicar la misma técnica de extracción de características para las señales relacionadas con la pronunciación de la vocal /o/. La tasa de clasificación promedio para la red entrenada y embebida fue de 
98.484\% y 99.126\% respectivamente, evidenciando una vez más la eficacia de la red al momento de realizar la separación de los grupos establecidos. Después de verificar la clasificación realizada por la red, y teniendo claro el orden en el que están las categorías, la tarea de identificación se reduce a encontrar cuál de las tres de neuronas de salida tiene el mayor valor, por ejemplo, si la respuesta de la red ante un patrón nuevo tiene su mayor valor en la posición 2, se afirma que el patrón está asociado a la pronunciación de la vocal /e/.

Tabla 3. Porcentajes de pertenencia para nuevas muestras de la vocal /o/

\begin{tabular}{|c|c|c|c|c|c|c|}
\hline \multirow{2}{*}{ \# Muestra } & \multicolumn{3}{|c|}{$\begin{array}{l}\text { Porcentaje de pertenencia con la } \\
\text { red neuronal en el computador (\%) }\end{array}$} & \multicolumn{3}{|c|}{$\begin{array}{l}\text { Porcentaje de pertenencia con la } \\
\text { red neuronal embebida (\%) }\end{array}$} \\
\hline & $\begin{array}{l}\text { Clase 1: } \\
\text { Vocal /a/ }\end{array}$ & $\begin{array}{l}\text { Clase } 2 \text { : } \\
\text { Vocal /e/ }\end{array}$ & $\begin{array}{l}\text { Clase 3: } \\
\text { Vocal /o/ }\end{array}$ & $\begin{array}{l}\text { Clase 1: } \\
\text { Vocal /a/ }\end{array}$ & $\begin{array}{l}\text { Clase } 2 \text { : } \\
\text { Vocal /e/ }\end{array}$ & $\begin{array}{l}\text { Clase 3: } \\
\text { Vocal /o/ }\end{array}$ \\
\hline 1 & $1,07 \times 10^{-07}$ & $2,66 \times 10^{-06}$ & 97,23 & $2,17 \times 10^{-07}$ & $1,52 \times 10^{-06}$ & 98,27 \\
\hline 2 & $1,59 \times 10^{-07}$ & $1,43 \times 10^{-06}$ & 98,42 & $1,32 \times 10^{-07}$ & $7,71 \times 10^{-09}$ & 99,82 \\
\hline 3 & $1,09 \times 10^{-09}$ & $4,49 \times 10^{-09}$ & 94,42 & $2,26 \times 10^{-07}$ & $5,75 \times 10^{-07}$ & 99,52 \\
\hline 4 & $8,27 \times 10^{-06}$ & $7,47 \times 10^{-08}$ & 96,52 & $1,97 \times 10^{-07}$ & $4,31 \times 10^{-09}$ & 99,82 \\
\hline 5 & $4,41 \times 10^{-08}$ & $8,68 \times 10^{-08}$ & 99,87 & $2,11 \times 10^{-07}$ & $3,83 \times 10^{-09}$ & 99,76 \\
\hline 6 & $3,08 \times 10^{-07}$ & $1,94 \times 10^{-07}$ & 99,50 & $8,05 \times 10^{-08}$ & $4,18 \times 10^{-09}$ & 99,45 \\
\hline 7 & $2,95 \times 10^{-07}$ & $7,26 \times 10^{-07}$ & 98,98 & $9,12 \times 10^{-08}$ & $3,24 \times 10^{-08}$ & 99,21 \\
\hline 8 & $1,95 \times 10^{-08}$ & $3,57 \times 10^{-09}$ & 99,98 & $4,53 \times 10^{-04}$ & $4,78 \times 10^{-06}$ & 98,25 \\
\hline 9 & $1,37 \times 10^{-08}$ & $1,01 \times 10^{-08}$ & 99,98 & $2,15 \times 10^{-07}$ & $7,42 \times 10^{-08}$ & 98,74 \\
\hline 10 & $2,01 \times 10^{-08}$ & $3,72 \times 10^{-08}$ & 99,94 & $1,56 \times 10^{-07}$ & $3,65 \times 10^{-07}$ & 98,42 \\
\hline Promedio & $9,24 \times 10^{-07}$ & $5,23 \times 10^{-07}$ & 98,484 & $4,55 \times 10^{-05}$ & $3,65 \times 10^{-07}$ & 99,126 \\
\hline
\end{tabular}

\section{CONCLUSIONES}

Las redes neuronales son algoritmos que se usan para una multitud de fines, como el control de sistemas, regresiones no lineales o clasificación e identificación de patrones, como se realizó en este trabajo. Este tipo de algoritmos resulta altamente costoso a nivel computacional en el momento del entrenamiento, dependiendo de los patrones y la cantidad de clases a separar. Sin embargo, una vez se han encontrado los parámetros o pesos, que permiten agrupar los patrones de acuerdo a sus características con un error de clasificación mínimo, se puede realizar el almacenamiento de los mismos para su posterior uso sin la necesidad de realizar un re-entrenamiento de la red para reconocer nuevas muestras.

El uso de sistemas embebidos para la implementación de algoritmos de inteligencia artificial, como las redes neuronales, permite elaborar aplicaciones más versátiles al convertir dicho algoritmo, en un algoritmo portable, además la capacidad de procesamiento de la red neuronal, funcionando en el sistema embebido fue muy similar al que tendría en un ordenador, como se demostró en este documento.

\section{AGRADECIMIENTOS}

A la vicerrectoría de investigaciones de la Universidad Militar Nueva Granada, por la financiación del proyecto ING/INV 1762 titulado "Dispositivo reproductor de voz del lenguaje español a través de habla subvocal e interfaz cerebro-computador", año 2015.

\section{REFERENCIAS}

Adam, T. B., Salam, M. S., y Gunawan, T. S. Wavelet based Cepstral Coefficients for neural network speech recognition, http://doi.org/10.1109/ICSIPA.2013.6708048, In 2013 IEEE International Conference on Signal and Image Processing Applications , 447-451, (2013)

Baek, S.-M., Park, J.-W., y Venayagamoorthy, G. K. Power System Control With an Embedded Neural Network in Hybrid System Modeling, http://doi.org/10.1109/TIA.2008.2002172, IEEE Transactions on Industry Applications, 44(5), 1458-1465, (2008)

Bao, J., Chen, Y., y Yu, J. An optimized discrete neural network in embedded systems for road recognition, http://doi.org/10.1016/j.engappai.2012.01.016, Engineering Applications of Artificial Intelligence, 25(4), 775782, (2012) 
Brandão, A. S., Cataldo, E., y Leta, F. R, Método de Línea de Transmisión aplicado a la Acústica del Tracto Vocal a través de un Modelo 3D Reconstruido, http://doi.org/10.4067/S0718-07642012000200018, Información Tecnológica, 23(2), 167-180, (2012)

Chiang, K.-W., Huang, Y.-W., Li, C.-Y., y Chang, H.-W. An ANN embedded RTS smoother for an INS/GPS integrated Positioning and Orientation System, http://doi.org/10.1016/j.asoc.2010.10.011, Applied Soft Computing, 11(2), 2633-2644, (2011)

Chih-Lyang Hwang, Chien-Wu Lan, y Ying-Jer Chou. "Search, Track, and Kick to Virtual Target Point" of Humanoid Robots by a Neural-Network-Based Active Embedded Vision System. http://doi.org/10.1109/JSYST.2013.2280852, IEEE Systems Journal, 9(1), 107-118, (2015)

Fu, Q., Zhao, Y., y Juang, B.-H. Automatic Speech Recognition Based on Non-Uniform Error Criteria. IEEE Transactions on Audio, http://doi.org/10.1109/TASL.2011.2165279, Speech, and Language Processing, 20(3), 780-793, (2012)

Gärdenfors, P., y Warglien, M. The Development of Semantic Space for Pointing and Verbal Communication, The Construal of Spatial Meaning: Windows Into Conceptual Space, $1^{\text {a }}$ edición, 29-42, Oxford University Press, Oxford, Inglaterra, (2013)

Knapp, M., Hall, J., y Horgan, T. Nonverbal Communication in Human Interaction, $8^{a}$ edición, 359-387, Wadsworth Cengage Learning, Boston, USA, (2013)

Kuamr, A., Dua, M., y Choudhary, T. Continuous Hindi speech recognition using Gaussian mixture HMM. http://doi.org/10.1109/SCEECS.2014.6804519, In 2014 IEEE Students' Conference on Electrical, Electronics and Computer Science, 1-5, (2014)

Kuan, T.-W., Wang, J.-F., y Shang-Hung, T. Optimized radix-2 FFT and Mel-filter bank in MFCC-based events sound recognition chip design for active smart warming care. http://doi.org/10.1109/ICOT.2014.6956633, In 2014 International Conference on Orange Technologies, 1, 197-200, (2014)

Lu, B., Wu, J., Wang, Y., y Li, J, A speech recognition system based on multiple neural networks. http://doi.org/10.1109/ICNC.2010.5583132, In 2010 Sixth International Conference on Natural Computation, 1, 48-51, (2010)

Martínez, A., Pineda, Z., y Ramos, A. Ocultamiento del Mensaje de Señales de Voz usando la Transformada de Ondita Haar Discreta. http://doi.org/10.4067/S0718-07642010000300015, Información Tecnológica, 21(3), 135-140, (2010)

Mella, M. R., y Soto-Barba, J, http://www.redalyc.org/articulo.oa?id=134516549004, ISSN: 0717-1285, Timbre vocálico en hablantes de español como segunda lengua. Onomázein, 1(11), 57-65. (2005)

Mitra, V., Franco, H., Graciarena, M., y Vergyri, D. Medium-duration modulation cepstral feature for robust speech recognition. http://doi.org/10.1109/ICASSP.2014.6853898. In 2014 IEEE International Conference on Acoustics, Speech and Signal Processing (ICASSP) ,1749-1753, (2014)

Moreno, R. G., Martínez, M. A., Nakano, M., y Pérez, H. M. Desarrollo de Algoritmos para Muestreo Compresivo Aplicado a Señales de Audio. http://doi.org/10.4067/S0718-07642014000500019. Información Tecnológica, 25(5), 137-146. (2014)

Siniscalchi, S. M., Svendsen, T., y Lee, C.-H. An artificial neural network approach to automatic speech processing. http://doi.org/10.1016/j.neucom.2014.03.005. Neurocomputing, 140, 326-338. (2014)

Ting, H.-N., Yong, B.-F., y Mirhassani, S. M. Self-Adjustable Neural Network for speech recognition. http://doi.org/10.1016/j.engappai.2013.06.004, Engineering Applications of Artificial Intelligence, 26(9), 2022-2027, (2013)

Valverde, A., y Soto-Barba, J, Variación del F1 y del F2 en hablantes cuyo F0 es grave o agudo en vocales del español de Chile acentuadas e inacentuadas, http://www.nuevarevistadelpacifico.cl/index.php/NRP/article/view/37, ISSN: 0719-5176, Nueva Revista del Pacífico, 61, 99-121. (2015)

Yoshioka, T., Sehr, A., Delcroix, M., Kinoshita, K., Maas, R., Nakatani, T., y Kellermann, W. Making Machines Understand Us in Reverberant Rooms: Robustness Against Reverberation for Automatic Speech 
Recognition. http://doi.org/10.1109/MSP.2012.2205029. IEEE Signal Processing Magazine, 29(6), 114-126, (2012)

Yuan, X.-C., Pun, C.-M., y Philip Chen, C. L, Robust Mel-Frequency Cepstral coefficients feature detection and dual-tree complex wavelet transform for digital audio watermarking, http://doi.org/10.1016/j.ins.2014.11.040, Information Sciences, 298, 159-179, (2015)

Yusnita, M. A., Paulraj, M. P., Yaacob, S., Bakar, S. A., y Saidatul, A. Malaysian English accents identification using LPC and formant analysis, http://doi.org/10.1109/ICCSCE.2011.6190572, In 2011 IEEE International Conference on Control System, Computing and Engineering, 472-476, (2011) 